\title{
Thin film models with constant source: model selection in a stochastic setting
}

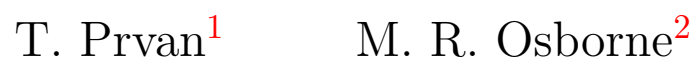

(Received 30 July 2007; revised 14 January 2008)

\begin{abstract}
Dunn and Tichenor [Atmospheric Environment, 22:885-894, 1988] proposed a class of differential equation models to describe the phenomenon of transient sink behaviour for organic emissions exhibited by interior surface films in state-of-the-art emission test chambers. The proposed model selection scheme embeds the derived models within a class of stochastic differential equations. The quality of model fit varies inversely with the strength of the stochastic forcing term; that is, if the model is adequate the stochastic forcing term should be small. Data from a particular application where the source can be considered to be constant demonstrates the approach. The approach can be applied to any phenomenon that is modelled by a class of linear differential equations where different models are embedded within a full model.
\end{abstract}

See http://anziamj.austms.org.au/ojs/index.php/ANZIAMJ/article/view/344 for this article, (C) Austral. Mathematical Soc. 2008. Published January 23, 2008. ISSN 1446-8735 


\section{Contents}

1 Introduction

C393

2 General form of thin film models

C394

3 Thin film models in a stochastic setting

C396

3.1 Stochastic formulation of smoothing spline . . . . . . . C C396

3.2 Stochastic formulation of thin film models . . . . . . . C398

4 Moth crystal cake example

C402

5 Conclusion

C404

References

C404

\section{Introduction}

Interior surface films in state-of-the-art emission chambers can act as a transient sink for organic emissions according to Dunn and Tichenor [3]. They developed a class of differential equations to model this phenomenon. Within their setting they have a full model, sink model, vapour pressure model, and dilution model. The sink model, vapour pressure model and dilution model are embedded within the full model. This work is outlined in Section 2. Dunn and Tichenor choose the 'best' model on the basis of the model which has the smallest residual sum of squares.

Section 3 places the class of differential equations proposed by Dunn and Tichenor [3] within a stochastic setting by extending the work of Osborne and Prvan [4]. The stochastic forcing term is used to choose the 'best' model since the models are embedded. The quality of the model fit varies inversely with the strength of the stochastic forcing term; that is, if the model is adequate 


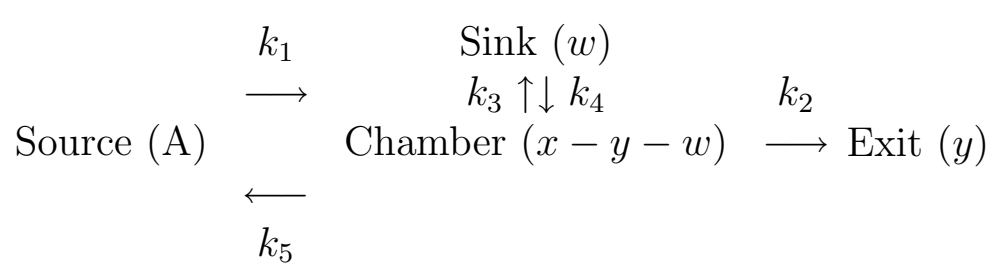

Figure 1: Flow among the compartments.

the stochastic forcing term should be small. Within this framework it is possible to come up with several 'best' models; if this happens, the simplest 'best' model is chosen, that is, the model with the least parameters.

This article demonstrates how the best model is chosen within this framework on the basis of the stochastic forcing term. In Section 4 experimental data from a particular application where the source is considered to be constant is used to demonstrate the approach and the results are discussed.

\section{General form of thin film models}

According to Dunn and Tichenor [3], interior surface films in state-of-theart emission chambers act as a transient sink for organic emissions. A class of differential equations was developed by them to model this phenomenon. Within their setting it is possible to consider four classes of models: full, sink, vapour pressure and dilution.

Dunn and Tichenor [3] postulate a test chamber system consisting of four mathematical compartments: the source, the well mixed chamber contents, an exit, and a sink. The source is a film source which may be decreasing (for example carpet glue on an inert carrier) or constant (for example a cake of moth crystal). The diagram in Figure 1 illustrates the rate constants that describe the flow among the compartments. Here A is the initial mass to 
be emitted by the source, $x=x(t)$ is the mass emitted to the chamber by time $t, y=y(t)$ is the mass exiting the chamber by time $t$, and $w=w(t)$ is the mass in the sink at time $t$. The concentration in the chamber at time $t$ is $C(t)=(x-y-w) / V$ where $V$ is the chamber volume. As in practice the concentration in the chamber is observed at different times $t$.

In a well mixed chamber Dunn and Tichenor [3] state that the system is adequately described by the following set of ordinary differential equations

$$
\begin{aligned}
\frac{d x}{d t} & =k_{1} g(x, t)-k_{5}(x-y-w), \\
\frac{d y}{d t} & =k_{2}(x-y-w), \\
\frac{d w}{d t} & =k_{3}(x-y-w)-k_{4} w .
\end{aligned}
$$

The right hand side of Equation (1) defines the emission rate of the source at time $t$. According to Equation (1) the rate of introduction of material into the chamber is proportional to $g(x, t)$ which is a function of time and the amount already emitted, while being inhibited by the mass in the chamber, or equivalently, chamber concentration since the chamber volume is constant. The function $g(x, t)$ describes the potential for emissions from the source into the chamber. According to Equation (2) the emission rate from the chamber is proportional to the amount of mass (or concentration) in the chamber. Equation (3) states that the rate at which material moves to the sink is proportional to the mass (or concentration) in the chamber, while the rate of removal from the sink is proportional to the amount already in the sink. The air flow rate is fixed during the experiment. Assuming a constant air flow rate $F$ through the chamber we have that $k_{2}=F / V$, the number of air changes in unit time. The full set of models possible in this setting are summarized in Table 1.

For constant source models the source acts as if it were a constant emitter for a finite period of time. A particular example given by Dunn and Tichenor [3] is the moth crystal cake. The emission rate of the moth crystal 
TABLE 1: Full set of models.

\begin{tabular}{lll} 
Models & & \\
\hline Full & $k_{3} \neq 0$ & $k_{5} \neq 0$ \\
Sink & $k_{3} \neq 0$ & $k_{5}=0$ \\
Vapour pressure & $k_{3}=0$ & $k_{5} \neq 0$ \\
Dilution & $k_{3}=0$ & $k_{5}=0$ \\
\hline
\end{tabular}

cake is limited by its surface area so is a good approximation to a source which acts as a constant emitter. Effectively, for a finite period of time, the source tends to be infinite and if the potential for emissions is constant during the time period considered then $g(x, t)=1$. Section 4 looks at their test labelled 10.1.

\section{Thin film models in a stochastic setting}

We first look briefly at the stochastic formulation of the smoothing spline and then extend this to fit thin film models in a stochastic setting.

\subsection{Stochastic formulation of smoothing spline}

Suppose that the data $\left(t_{1}, z_{1}\right), \ldots,\left(t_{n}, z_{n}\right)$ are given and it is assumed that the data is decomposed as a signal plus noise model:

$$
z_{i}=f\left(t_{i}\right)+\epsilon_{i}, \quad \epsilon_{i} \sim N\left(0, \sigma^{2}\right), \quad i=1, \ldots, n .
$$


We could use a smoothing spline to fit the 'signal' $f(t)$. A smoothing spline $f$ is the minimizer of

$$
\frac{1}{n} \sum_{i=1}^{n}\left(z_{i}-f\left(t_{i}\right)\right)^{2}+\mu \int_{t_{1}}^{t_{n}}\left(f^{(m)}(t)\right)^{2} d t .
$$

The resultant curve is a piecewise polynomial of degree $2 m-1$ with $2 m-2$ continuous derivatives.

Wahba [7] modelled the signal by the stochastic differential equation

$$
\frac{d^{m} \mathrm{x}}{d t^{m}}=\sigma \sqrt{\lambda} \frac{d \omega}{d t}
$$

where $\omega(t)$ is a Wiener process with unit dispersion parameter and smoothing parameter $\lambda=1 / \mu$. Let $\mathbf{x}\left(t_{1}\right)=\left[\begin{array}{llll}\mathrm{x}\left(t_{1}\right) & \mathrm{x}^{\prime}\left(t_{1}\right) & \cdots & \mathrm{x}^{(m-1)}\left(t_{1}\right)\end{array}\right]^{T}$ be the vector of initial conditions on (6). Wahba shows that if $\mathbf{x}\left(t_{1}\right)$ has a diffuse prior distribution; that is $\mathbf{x}\left(t_{1}\right) \sim N\left(\mathbf{0}, \gamma^{2} I_{m}\right)$ and letting $\gamma^{2} \rightarrow \infty$, then

$$
f(t)=\lim _{\gamma \rightarrow \infty} E\left\{\mathrm{x}(t) \mid z_{1}, \ldots, z_{n}\right\}
$$

where $E\left\{\mathrm{x}(t) \mid z_{1}, \ldots, z_{n}\right\}$ is the conditional expectation of $\mathrm{x}(t)$ given the data $z_{1}, \ldots, z_{n}$.

Wecker and Ansley [9] presented a stochastic formulation of a polynomial smoothing spline using this result.

The stochastic differential equation (6) is written as

$$
\frac{d \mathbf{x}}{d t}=\left(\begin{array}{cc}
\mathbf{0}_{m-1} & I_{m-1} \\
0 & \mathbf{0}^{T}
\end{array}\right) \boldsymbol{x}+\sigma \sqrt{\lambda} \frac{d \omega}{d t} \boldsymbol{e}_{m}
$$

and the observation equation (4) as

$$
z\left(t_{i}\right)=\boldsymbol{e}_{1}^{T} \mathbf{x}\left(t_{i}\right)+\epsilon_{i},
$$


where $\boldsymbol{e}_{1}$ is a vector with 1 in the first position and zeroes elsewhere.

For given $\lambda$ the Kalman filter is used to compute $\mathbf{x}\left(t_{i} \mid i\right)$, the conditional expectation of $\mathbf{x}\left(t_{i}\right)$ given observations $z_{1}, z_{2}, \ldots, z_{i}$ and $\mathbf{x}\left(t_{i+1} \mid i\right)$, the conditional expectation of $\mathbf{x}\left(t_{i+1}\right)$ given observations $z_{1}, z_{2}, \ldots, z_{i}$. These quantities and their associated variances are needed to calculate $\mathbf{x}\left(t_{i} \mid n\right)$, the conditional expectation of $\mathbf{x}\left(t_{i}\right)$ given all the observations $z_{1}, z_{2}, \ldots, z_{n}$. To initiate the Kalman filter we use $\mathbf{x}\left(t_{1} \mid 0\right)=\mathbf{0}$ (an estimate of $\mathbf{x}\left(t_{1}\right)$ given no data) and place a diffuse prior on it. To do this we let the variance of $\mathbf{x}\left(t_{1} \mid 0\right)$ be $S_{1 \mid 0}=\gamma^{2} I_{p}$ where $\gamma \rightarrow \infty$. In practice we set $\gamma$ to be large and it was shown by Osborne and Prvan [4] that the effect of the diffuse prior disappears by the $m$ th step. The smoother developed by Rauch, Tung and Striebel [6], which we will refer to as the RTS Smoother, is used to compute $\mathbf{x}\left(t_{i} \mid n\right)$. For $t_{i-1} \leq t \leq t_{i}$ the interpolation smoother is used to compute $\mathbf{x}(t \mid n)$ and the first entry will be the point estimate of the signal $f$ at point $t$, the smoothing spline evaluated at time $t$. Ansley and Kohn [2] provide a geometrical derivation of the Interpolation Smoother whereas Anderson and Moore [1] provide the more traditional derivation. Maximum Likelihood Estimation or Generalised Cross Validation can be used to find the smoothing parameter $\lambda$. Wahba [8] compared these methods.

\subsection{Stochastic formulation of thin film models}

Osborne and Prvan [4] considered a generalisation of the stochastic formulation of smoothing splines presented by Wecker and Ansley [9]. Data $\left(t_{1}, z_{1}\right), \ldots,\left(t_{n}, z_{n}\right)$ are given and the observed $z_{i}$ at time $t_{i}$ is assumed to be decomposed as the signal plus noise model

$$
z_{i}=\boldsymbol{h}^{T} \mathbf{x}\left(t_{i}\right)+\epsilon_{i}, \quad \epsilon_{i} \sim N\left(0, \sigma^{2}\right), \quad i=1, \ldots, n,
$$

where $\boldsymbol{h}, \mathbf{x}\left(t_{i}\right) \in \mathbb{R}^{m}$ and $y_{i}, \epsilon_{i} \in \mathbb{R}$. We are only considering $\boldsymbol{h}$ constant. The errors $\epsilon_{i}$ are assumed to independent and $\mathbf{x}(t)$ satisfies the stochastic 
differential equation

$$
\frac{d \mathbf{x}}{d t}=M \mathbf{x}+\sigma \sqrt{\lambda} \frac{d \boldsymbol{\omega}}{d t},
$$

where $M: \mathbb{R}^{m} \rightarrow \mathbb{R}^{m}, \boldsymbol{\omega}(t)$ is a $m$-dimensional Wiener process independent of $\epsilon_{i}, i=1,2, \ldots, n$, satisfying, for positive semi-definite $W: \mathbb{R}^{m} \rightarrow \mathbb{R}^{m}$ given,

$$
E\left\{(\boldsymbol{\omega}(t+\delta)-\boldsymbol{\omega}(t))(\boldsymbol{\omega}(t+\delta)-\boldsymbol{\omega}(t))^{T}\right\}=W \delta .
$$

The smoothing parameter $\lambda$ defines the relative scale of the two noise processes.

To place thin film models in a stochastic setting we generalise the stochastic differential equation for generalised smoothing splines (11) to

$$
\frac{d \mathbf{x}}{d t}=M \mathbf{x}+\boldsymbol{g}(t)+\sigma \sqrt{\lambda} \frac{d \boldsymbol{\omega}}{d t},
$$

where $M: \mathbb{R}^{m} \rightarrow \mathbb{R}^{m}$ and $\boldsymbol{\omega}(t)$ is a $m$-dimensional Wiener process as described above. The observation equation (10) remains the same.

We now obtain the state space formulation of the extended generalized smoothing spline. Let $X\left(t, t_{1}\right)$ be the fundamental matrix of the associated homogeneous differential equation. That is,

$$
d X\left(t, t_{1}\right) / d t=M X\left(t, t_{1}\right), \quad X\left(t_{1}, t_{1}\right)=I_{m} .
$$

The solution to the stochastic differential equation (13) satisfying $\mathbf{x}\left(t_{1}\right)=\mathbf{x}_{1}$ is

$$
\mathbf{x}(t)=X\left(t, t_{1}\right) \mathbf{x}_{1}+\int_{t_{1}}^{t} X(t, s) \boldsymbol{g}(s) d s+\sigma \sqrt{\lambda} \int_{t_{1}}^{t} X(t, s) \frac{d \boldsymbol{\omega}}{d s} d s .
$$

This solution is written in the form of a recursion relation as

$$
\mathbf{x}_{i+1}=X_{i+1} \mathbf{x}_{i}+\int_{t_{i}}^{t_{i+1}} X\left(t_{i+1}, s\right) \boldsymbol{g}(s) d s+\sigma \sqrt{\lambda} \boldsymbol{u}_{i+1},
$$


with $\mathbf{x}_{i}=\mathbf{x}\left(t_{i}\right), X_{i+1}=X\left(t_{i+1}, t_{i}\right)$ and $\boldsymbol{u}_{i+1}=\boldsymbol{u}\left(t_{i+1}, t_{i}\right)$ where $\boldsymbol{u}_{i+1}=$ $\int_{t_{i}}^{t_{i+1}} X\left(t_{i+1}, s\right) \frac{d \omega}{d s} d s$ which has a $N\left(\mathbf{0}, \Omega\left(t_{i+1}, t_{i}\right)\right)$ distribution with covariance matrix

$$
\Omega_{i+1}=\Omega\left(t_{i+1}, t_{i}\right)=\int_{t_{i}}^{t_{i+1}} X\left(t_{i+1}, s\right) W X\left(t_{i+1}, s\right)^{T} d s .
$$

Covariance matrices are by definition at least positive semi-definite so care is needed in choosing $W$ to ensure that this holds. This is why we have the restriction that $W$ be positive semi-definite.

We now have the state space formulation (10) and (16). We now use the Kalman filter for our particular state space formulation to compute $\mathbf{x}\left(t_{i} \mid\right.$ $i-1)$ and $\mathbf{x}\left(t_{i} \mid i\right)$. We place a diffuse prior on $\mathbf{x}\left(t_{1}\right)$ to utilise a generalisation of Wahba's result [7] where our point estimate is $\boldsymbol{h}^{T} \mathbf{x}(t \mid n)$. To initiate the Kalman filter we use $\mathbf{x}\left(t_{1} \mid 0\right)=\mathbf{0}$ and let the variance be $S_{1 \mid 0}=\gamma^{2} I_{p}$ where $\gamma \rightarrow \infty$ (that is, our diffuse prior on $\mathbf{x}(t)$ ). In practice we set $\gamma$ to be large. The RTS Smoother [6] computes $\boldsymbol{x}\left(t_{i} \mid n\right)$. For $t_{i-1} \leq t \leq t_{i}$ we use the interpolation smoother modified for state equation with forcing term (this is new) to compute $\mathbf{x}(t \mid n)$.

The forward pass of the Kalman filter and backward pass of the RTS Smoother for given model parameters need only be computed once for fixed $\lambda$. Maximum Likelihood Estimation or Generalized Cross Validation finds the smoothing parameter as well as the model parameters. A model is plausible if the smoothing parameter $\lambda$ is small, whereas for a large value of $\lambda$ it is not.

For reference the recursions are given below for our particular state space formulation.

Kalman Filter Initiate with $\mathbf{x}_{1 \mid 0}=\mathbf{0}$ and $S_{1 \mid 0}=\gamma^{2} I_{m}$.

$$
\begin{aligned}
& \mathbf{x}_{k+1 \mid k}=X_{k+1} \mathbf{x}_{k \mid k}+\int_{t_{k}}^{t_{k+1}} X\left(t_{k+1}, s\right) \boldsymbol{g}(s) d s, \\
& S_{k+1 \mid k}=X_{k+1} S_{k+1 \mid k} X_{k+1 \mid k}^{T}+\lambda \sigma^{2} \Omega\left(t_{k+1}, t_{k}\right),
\end{aligned}
$$




$$
\begin{aligned}
d_{k+1}= & \boldsymbol{h}^{T} S_{k+1 \mid k} \boldsymbol{h}+\sigma^{2} \\
\mathbf{x}_{k+1 \mid k+1}= & \mathbf{x}_{k+1 \mid k}+S_{k+1 \mid k} \boldsymbol{h} d_{k+1}^{-1}\left(y_{k+1}-\boldsymbol{h}^{T} \mathbf{x}_{k+1 \mid k}\right), \\
S_{k+1 \mid k}= & S_{k+1 \mid k}-S_{k+1 \mid k} \boldsymbol{h} d_{k+1}^{-1} \boldsymbol{h}^{T} S_{k+1 \mid k}^{T}, \\
& k=1, \ldots, n-1 .
\end{aligned}
$$

RTS Smoother Initiate with $\mathbf{x}_{n \mid n}$ obtained from a forward pass of the Kalman Filter.

$$
\mathbf{x}_{k \mid n}=\mathbf{x}_{k \mid k}+S_{k \mid k} X_{k+1}^{T} S_{k+1 \mid k}^{-1}\left(\mathbf{x}_{k+1 \mid n}-\mathbf{x}_{k+1 \mid k}\right), \quad k=n-1, \ldots, 1 .
$$

Interpolation Smoother For $t_{i-1} \leq t<t_{i}$,

$$
\mathbf{x}(t \mid n)=X\left(t, t_{i-1}\right) \mathbf{x}_{i-1 \mid i-1}+\int_{t_{i-1}}^{t} X(t, s) \boldsymbol{g}(s) d s+A 1\left(t_{i}, t\right)\left(\mathbf{x}_{i \mid n}-\mathbf{x}_{i \mid i-1}\right),
$$

where

$$
A 1\left(t_{i}, t\right)=\left[X\left(t, t_{i-1}\right) S_{i-1 \mid i-1} X_{i}^{T}+\Omega\left(t, t_{i-1}\right) X\left(t_{i}, t\right)^{T}\right] S_{i \mid i-1}^{-1} .
$$

For the thin film models proposed by Dunn and Tichenor [3] we have

$$
\begin{aligned}
\mathbf{x}^{T} & =\left[\begin{array}{lll}
x & y & w
\end{array}\right], \\
M & =\left[\begin{array}{rrr}
-k_{5} & k_{5} & k_{5} \\
k_{2} & -k_{2} & -k_{2} \\
k_{3} & -k_{3} & -\left(k_{3}+k_{4}\right)
\end{array}\right], \\
\boldsymbol{g}(t)^{T} & =\left[\begin{array}{lll}
k_{1} g(x, t) & 0 & 0
\end{array}\right], \\
\boldsymbol{h}^{T} & =\left[\begin{array}{lll}
1 & -1 & -1
\end{array}\right] .
\end{aligned}
$$

For constant source we have $g(x, t)=1$ so $\boldsymbol{g}(t)=k_{1} \boldsymbol{e}_{1}$. To ensure that the covariance matrices associated with the state transition equation (16) are 
positive definite we choose $W=X\left(t_{i+1}, s\right)^{-1}\left(X\left(t_{i+1}, s\right)^{T}\right)^{-1}$ in (17) to give

$$
\Omega_{i+1}=\Omega\left(t_{i+1}, t_{i}\right)=\int_{t_{i}}^{t_{i+1}} I_{m} d s=\left(t_{i+1}-t_{i}\right) I_{m} .
$$

With this choice we also have the state transition covariance matrix being model parameter free.

\section{Moth crystal cake example}

Dunn and Tichenor [3] present results for 13 emissions tests of moth crystal cakes. The chamber was cleaned between each test. They fitted the four constant source models to each data set using non linear least squares to determine the unknown parameters in the experiment like $k_{1}$. The parameter $k_{2}$ is fixed by the experiment and is the the number of air changes per hour. They had $V=166 l$. The criteria they use to determine the best model for each test is to choose the model that produces the smallest residual sum of squares. We look at the test labelled 10.1 in more detail.

The four models were fitted to the data from the test labelled 10.1. Maximum likelihood estimation obtained the parameter estimates as well as the smoothing parameter. We dealt with the diffuse prior explicitly setting $S_{1 \mid 0}=\gamma^{2} I_{3}$ where $\gamma=1000$. Details on how to do this are given by Prvan and Osborne [5]. Each model used as its first starting value the parameters (excluding smoothing parameter) obtained from the nonlinear least squares fits to the data. Different values of $\lambda$ were tried in the initial estimates. Matlab 7.1 was used for the programming and the Optimisation Toolbox function fmincon was used to find the parameter values using the negative of the partial log likelihood as the function to be minimised.

We only searched for the smoothing parameter in the interval $\left[10^{-6}, 10^{6}\right]$. All four models fitted had a smoothing parameter value of $10^{-6}$ and the 


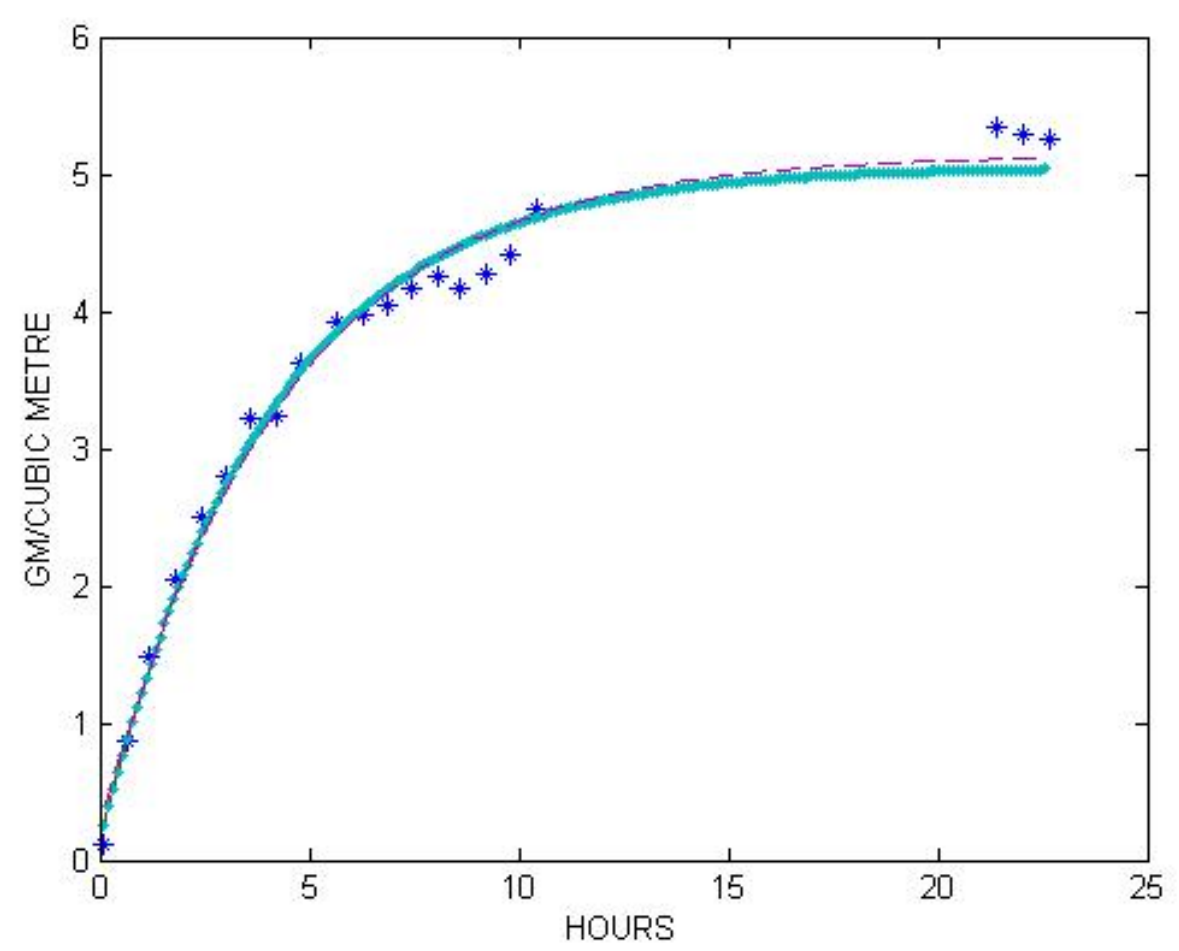

FIGURE 2: Extended generalized smoothing spline fits for full model, vapour pressure model, sink model and dilution model fitted to 1,4-Dichlorobenzene emission from moth crystal. The full model, vapour pressure model and sink model fit are the same solid line, and the dashed line is the dilution model fit. 
resulting fits are shown in Figure 2. The resulting fits to the data shows that the simplest model, the dilution model, is adequate.

\section{Conclusion}

The approach presented here can be applied to any linear system of differential equations used to model an application which has different embedded candidate models. If the full model fitted to the application data does not have a small smoothing parameter then the linear system of differential equations is not a good model for the application. Full model here refers to the model with the most parameters being considered. Reduced models are those obtained by setting some of the parameters in the full model to zero. If the full model fitted to the data does have a small smoothing parameter, then we can investigate further to see if a reduced model will suffice. A reduced model is considered to be a candidate model if the smoothing parameter is small when the reduced model is fitted to the data. This approach is analogous to backward stepwise regression in statistics but instead of basing our decision on the reduction in the residual sum of squares we base it on whether the smoothing parameter is small.

\section{References}

[1] B. D. Anderson and J. B. Moore Optimal Filtering, Prentice-Hall, Inc., Englewood Cliffs, N. J., 1979. C398

[2] C. F. Ansley and R. Kohn A geometrical derivation of the fixed interval smoothing algorithm. Biometrika, 69:486-487. C398 
[3] J. E. Dunn and B. A. Tichenor. Compensating for sink effects in emissions test chambers by mathematical modeling. Atmospheric Environment, 22:885-894, 1988. C393, C394, C395, C401, C402

[4] M. R. Osborne and T. Prvan. Smoothness and conditioning in generalised smoothing spline calculations. J. Austral. Math. Soc. Ser. B, 30:43-56, 1988. C393, C398

[5] T. Prvan and M. R. Osborne. Model selection in a stochastic setting. ANZIAM J., 45(E) ppC787-C799, 2004.

http://anziamj . austms . org. au/V45/CTAC2003/Prva/home.html C402

[6] H. E. Rauch, F. Tung and C. T. Striebel. Maximum Likelihood Estimates of Linear Dynamic Systems. AIAA J., 3: 1445-1450, 1965. C398, C400

[7] G. Wahba. Improper priors, spline smoothing and the problem of guarding against model errors in regression. J. R. Statist. Assoc., 40:364-372, 1978. C397, C400

[8] G. Wahba. A comparison of GCV and GML for choosing the smoothing parameter in the generalised spline smoothing problem. Ann. Statist., 13: 1378-1402, 1985. C398

[9] W. Wecker and C. F. Ansley. Signal extraction approach to non linear regression and spline smoothing. J. Amer. Statist. Assoc., 78: 81-89, 1983. C397, C398 


\section{Author addresses}

1. T. Prvan, Dept. of Statistics, Macquarie University, Sydney, Australia.

mailto:tprvan@efs.mq.edu.au

2. M. R. Osborne, Mathematical Sciences Institute, ANU, Australia. 